\title{
Low-dose quinine for treatment of chloroquine-resistant falciparum malaria in Sudanese pregnant women
}

I. Adam, ${ }^{1}$ M.H. Ibrahim, ${ }^{1}$ I.A. Alelbasit ${ }^{2}$ and M.I. Elbashir ${ }^{2}$

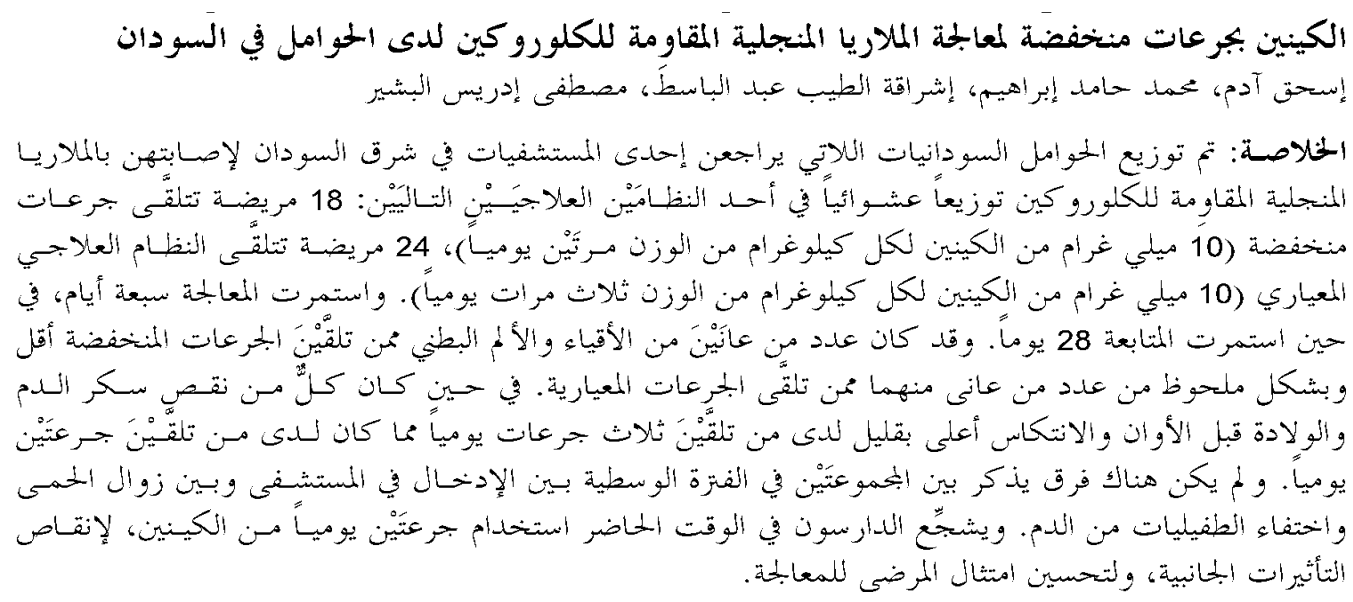

ABSTRACT Pregnant Sudanese women who presented at a hospital in eastern Sudan with chloroquineresistant falciparum malaria were randomly allocated to one of two quinine regimens: low-dose $(10 \mathrm{mg} / \mathrm{kg} 2$ times/day) (18 patients) or standard (10 mg/kg 3 times/day) (24 patients). Treatment was for 7 days and follow-up for 28 days. Significantly fewer patients in the low-dose group reported vomiting and abdominal pain than the standard regimen group. Hypoglycaemia, preterm labour and recrudescence were slightly but not significantly higher in patients in the standard group than low-dose group. There were no significant differences between the groups in the mean time from admission to remission of fever and parasite clearance. We tentatively advocate the use of quinine 2 times/day to reduce side-effects and improve compliance.

\begin{abstract}
Quinine à faible dose pour le traitement du paludisme à falciparum chloroquino-résistant chez des femmes enceintes soudanaises

RÉSUMÉ Des femmes enceintes soudanaises atteintes de paludisme à falciparum chloroquino-résistant qui ont consulté dans un hôpital du Soudan oriental ont été réparties de manière aléatoire entre les deux schémas thérapeutiques de quinine suivants : faible dose, $10 \mathrm{mg} / \mathrm{kg} 2 \mathrm{fois} / \mathrm{jour}$ ( 18 patientes), ou standard, $10 \mathrm{mg} / \mathrm{kg} 3$ fois/jour (24 patientes). Le traitement durait 7 jours et le suivi 28 jours. Un nombre significativement moindre de patientes dans le groupe du traitement à faible dose a signalé des vomissements et des douleurs abdominales par rapport aux patientes recevant le traitement standard. L'hypoglycémie, le travail prématuré et la recrudescence étaient légèrement, mais non significativement, plus élevés chez les patientes du groupe du traitement standard que dans le groupe du traitement à faible dose. II n'y avait aucune différence significative entre les deux groupes pour ce qui est du temps moyen entre l'admission, la rémission de la fièvre et l'élimination du parasite. Nous recommandons provisoirement l'utilisation de la quinine deux fois par jour pour réduire les effets secondaires et améliorer l'observance du traitement.
\end{abstract}

${ }^{\top}$ New Halfa Hospital, New Halfa, Sudan.

${ }^{2}$ Faculty of Medicine, University of Khartoum, Khartoum, Sudan.

Received: 14/07/03; accepted: 28/10/03

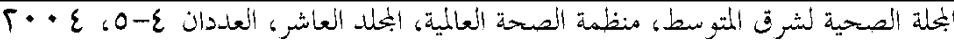




\section{Introduction}

In Sudan, falciparum malaria has been reported to cause a number of adverse maternal and fetal outcomes, such as maternal anaemia, low birth weight, preterm labour and perinatal mortality [1-6]. It is the leading cause of maternal mortality [5,6].

The treatment of falciparum infection remains the main means available to limit the impact of malaria on pregnancy [7]. The spread of chloroquine and sulfadoxine-pyrimethamine resistance in Sudan [810] necessitates the use of alternative drugs for the treatment of falciparum infections, such as quinine which is the second most prescribed antimalarial drug in Sudan [11]. Quinine is the treatment of choice for severe and chloroquine-resistant falciparum malaria.

The standard dose of quinine is $10 \mathrm{mg} /$ kg, 3 times per day for 7 days [12]. This dose has been reduced effectively in children without impairing its efficacy, and with marked improvement in compliance with drug use [13]. The side-effects of quinine treatment that can lead to poor compliance are tinnitus, nausea, vomiting, diarrhoea, hypoglycaemia and acute hypersensitivity. Physicians in central and eastern Sudan are now also giving quinine to pregnant women 2 times daily rather than 3 times, with apparently good outcomes. The present study was carried out to verify the efficacy and safety of this ongoing clinical practice.

\section{Methods}

We performed a prospective clinical trial in New Halfa Hospital in eastern Sudan from the period November 2002 to March 2003. After verbal consent, all pregnant women presenting with failure of chloroquine therapy for the treatment of falciparum malaria were successively enrolled to the study. Those with one or more manifestations of severe falciparum malaria were excluded [14].

For both groups, quinine (Laboratoires Renaudin, France) was given under strict supervision, first by intravenous infusion in $5 \%$ dextrose solution over 2-4 hours, and when the patient could tolerate it, therapy was continued orally in the form of tablets. For this phase of the study, patients were randomized into 2 treatment groups: quinine $10 \mathrm{mg} / \mathrm{kg} 2$ times/day for 7 days (BD group) and quinine $10 \mathrm{mg} / \mathrm{kg} 3$ times/day for 7 days (TDS group).

A detailed record was made for each patient, including: personal data, medical and obstetric history, physical examination and use of antimalarials in the 3 weeks before entry to the study. During the followup, all patients were asked daily about the expected side-effects of quinine (tinnitus, vomiting and abdominal pain). Axillary temperature was recorded every 8 hours until it fell to normal $\left(37.5^{\circ} \mathrm{C}\right)$, and then daily until day 7. All patients were kept in the hospital for at least 7 days and then followed up weekly in the antenatal clinic for 28 days.

\section{Laboratory investigations}

Using finger prick blood samples, thick and thin blood smears were prepared from each patient in both groups, stained with Giemsa (pH 7.0, diluted in phosphate-buffered saline) and counted against 200 white blood cells assuming that the number of cells is $6000 / \mathrm{mm}^{3}$ of blood. Thin blood films, fixed in methanol and Giemsa-stained were made when the parasite species was doubtful. The blood films were repeated every 8 hours until 2 consecutive films were negative, then daily until day 7 and then on days 14, 21 and 28. Haemoglobin concentration and capillary blood glucose level were determined on presentation. Capillary blood

بلملة الصحية لشرق المتو سط، منظمة الصحة العلمية، البحلد العاشر، العددان ع-ه، ع • • 
glucose was estimated 2 hours after administration of the drug.

All patients were resident in the same area during the follow-up period. Therefore, the possibility of re-infection or recrudescence could not be ruled out. Three spots of blood were taken on filter paper initially and later if parasites reappeared microscopically during the follow-up period. Primers from 3 polymorphic Plasmodium falciparum antigens; merozoite surface protein-1 and 2 (MSP-1 and MSP-2) and glutamate-rich protein (GLURP) were used in polymerase chain reaction analysis (PCR) to differentiate between true recrudescence and re-infection as described previously [15].

\section{Evaluation criteria}

The efficacy and side-effects of the 2 regimens of quinine were assessed according to parasite clearance time, fever clearance time, occurrence of side-effects (tinnitus, vomiting, abdominal pain and hypoglycaemia) and recrudescence. Parasite clearance time was defined as the time between start of treatment until 2 consecutive negative blood smears were obtained. Fever remission time was defined as the time between admission and achievement of normal body temperature.

\section{Statistics}

Data was entered into the computer using SPSS/PC batching for data analysis. Simple frequency distribution cross-tabulation, descriptive statistics, mean, $t$-test and chisquared with probability $\leq 0.05$ was used for testing the hypotheses.

\section{Ethics}

Informed consent was obtained from the women who participated in the study. Ethical clearance for the study was obtained from the Faculty Research Board, Faculty of Medicine, University of Khartoum and the National Ethical Committee at the Sudanese Federal Ministry of Health.

\section{Results}

Sixty-five pregnant women presented to New Halfa hospital with manifestations of chloroquine-resistant falciparum malaria during the study period. After confirmation of the infection, 14 patients were excluded from the study because they had severe manifestations of the disease. Initially, 25 patients were enrolled in the BD group, and 26 patients in the TDS group. However, 7/ 25 of the BD and 2/26 of the TDS group ( $P$ $=0.05$ ) were excluded from the follow-up and evaluation as they chose to leave hospital and continue the treatment at home after the first or the second dose of quinine.

Table 1 shows the major characteristics of the remaining women on presentation. There were no significant differences between the 2 groups in age, parity, weight, temperature, haemoglobin level, parasite count and random blood glucose level at presentation.

A slightly higher proportion of women in the $\mathrm{BD}$ group presented with vomiting than the TDS group-4/18 (22.2\%) versus $3 / 24$ (12.5\%) - but this was not statistically significant. On day 1 , vomiting was recorded in more BD patients; 7/18 (38.9\%) versus $4 / 24(16.7 \%)$ but this was not statistically significant $(P>0.05)$. On day 2 , significantly fewer patients in the BD than the TDS suffered from vomiting; 29/18 (50.0\%) versus $9 / 24$ (79.2\%) $(P<0.05)$.

Tinnitus was reported slightly less frequently by patients who received quinine $\mathrm{BD}$, than in those who received it TDS; $12 /$ $18(66.7 \%)$ versus 19/24 (79.2\%) $(P>$ $0.05)$. Significantly fewer patients reported abdominal pain in the BD group than in the

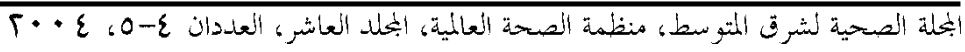




\begin{tabular}{|c|c|c|c|c|c|}
\hline \multirow[t]{2}{*}{ Variable } & \multicolumn{2}{|c|}{$\begin{array}{c}\text { BD regimen } \\
\quad(n=18)\end{array}$} & \multicolumn{2}{|c|}{$\begin{array}{c}\text { TDS regimen } \\
(n=24)\end{array}$} & \multirow[t]{2}{*}{$P$-value } \\
\hline & Mean & $S D$ & Mean & $S D$ & \\
\hline \multicolumn{6}{|l|}{ On admission } \\
\hline Age (years) & 25.2 & 6.0 & 25.5 & 6.9 & 0.4 \\
\hline Parity (No.) & 2.6 & 1.9 & 2.6 & 2.2 & 0.3 \\
\hline Weight $(\mathrm{kg})$ & 59.3 & 15.9 & 52.2 & 9.3 & 0.09 \\
\hline Gestational age (weeks) & 26.1 & 9.9 & 26.1 & 8.9 & 0.4 \\
\hline Axillary temperature $\left({ }^{\circ} \mathrm{C}\right)$ & 38.1 & 0.9 & 37.8 & 1.0 & 0.8 \\
\hline Haemoglobin level (g/L) & 89.0 & 5.6 & 86.0 & 9.0 & 0.08 \\
\hline Parasite count (rings $/ \mu \mathrm{L})$ & 5837 & 8361 & 4207 & 12325 & 0.6 \\
\hline Random blood sugar level (mg/dL) & 117.6 & 30.1 & 106.5 & 25.7 & 0.9 \\
\hline \multicolumn{6}{|l|}{ Treatment outcomes } \\
\hline Fever remission time ${ }^{a}$ (hours) & 25.5 & 12.1 & 21.0 & 16.9 & 0.13 \\
\hline Parasite clearance time ${ }^{b}$ (hours) & 27.7 & 12.9 & 33.7 & 12.7 & 0.63 \\
\hline
\end{tabular}

a Fever remission time was defined as the time between admission and achievement of normal body temperature $\left(37.5^{\circ} \mathrm{C}\right)$.

${ }^{b}$ Time from admission and start of treatment until 2 consecutive negative blood smears were obtained.

$S D=$ standard deviation

TDS group, $1 / 18(5.6 \%)$ versus $7 / 24$ $(29.2 \%)(P=0.05)$. Some of the TDS group developed hypoglycaemia; $4 / 24$ (16.7\%), but this was not recorded in any patient in the BD group (0/18); this difference was not significant $(P>0.05)$.

While none of the patients in the BD delivered prematurely ( $<37$ weeks), $2 / 24$ (8.3\%) patients in the TDS group delivered prematurely at 29 and 30 weeks gestational age and their babies died immediately $(P>$ 0.05 ).

True recrudescence was confirmed by parasite genotyping on days 21 and 28 in 2/ $18(11.1 \%)$ patients among the BD group. They were successfully treated with sulfadoxine-pyrimethamine. There was no detectable parasitaemia during follow-up in the TDS group, but this difference was not significant $(P>0.5)$.
The mean (SD) parasite clearance time was lower in the BD than in the TDS group, but this did not reach the level of significance: 27.2 (12.9) versus 33.7 (12.7) hours.

\section{Discussion}

This is the first study of the efficacy of low-dose quinine in the treatment of chloroquine-resistant falciparum malaria during pregnancy in an area of high chloroquine resistance in eastern Sudan [8].

The study showed that significantly more patients chose to continue the quinine treatment at home in the $\mathrm{BD}$ than in the TDS group and this may reflect the simplicity of this regimen. Moreover, quinine side-effects were reported more frequently

لبحلة الصحية لشرق المثتوسط، منظمة الصحة العلمية، البحلد العاشر، العدذان ع-0، ع • • 
in the TDS group than in the BD group; significantly more patients suffered from vomiting and abdominal pain in the TDS group than in the $\mathrm{BD}$ group. Although these side-effects were more frequent in the TDS than in the BD group, they might be considered a subjective assessment of these 2 regimens of quinine. Nevertheless, even objective side-effects were more frequent in the TDS than in the BD group. For example, hypoglycaemia was seen in more of the patients in the TDS group than the BD group (16.7\% versus $0 \%$ ) although the different not significant. However, hypoglycaemia was reported in around 50\% of pregnant women at one stage or another of severe falciparum malaria treated with quinine 3 times/day for 7 days [16], therefore, hypoglycaemia may be dose related. In a recent study, a low dose of quinine was used in children in a community-based study but hypoglycaemia was not assessed [13].

Two patients in the TDS group delivered prematurely and their babies died immediately, but there was no premature delivery in the BD group. In a previous study where we were testing the efficacy of quinine 3 times/day in the treatment of severe falciparum malaria during pregnancy in central Sudan, 3/33 (9\%) patients delivered prematurely, and only 1 patient delivered during quinine therapy [4]. This comparison should be viewed with caution because in the previous study we used quinine for severe illness, while such patients were excluded in the present study. However, no preterm labour was reported by McGready et al. in 1998 [17]. Malaria can cause abortion and preterm labour as well, and in central Sudan it was found to be the leading cause of low birth weight as a result of preterm labour [3]. However, the oxytocic effect of quinine on the pregnant uterus cannot be excluded totally. Previously, quinine was used as a labour-inducing agent but in high doses [18].

The parasite clearance time was shorter but not significantly so in the BD group than in the TDS group. However, 2 patients $(11.1 \%)$ in the BD group showed true parasite recrudescence during the follow-up, compared with none of the patient in the TDS group. This difference was not statistically significant and it should be viewed with caution as it might be due to quinine resistance in this area of Sudan. Previously, we have observed quinine resistance by in vivo testing and it has been confirmed by in vitro testing in a nearby area $[8,19]$. We have previously shown $6 \%$ quinine resistance or re-infection during pregnancy in central Sudan [4].

In conclusion, quinine in a low-dose regimen of 2 times/day causes fewer subjective side-effects and therefore is likely to improve patient compliance than the standard 3 times/day regimen. It also has a lower risk of hypoglycaemia (which is important in the outpatient setting) and of preterm labour. Its disadvantage is the higher probability of recrudescence, which is important in the light of emerging resistance in Africa, where the drug is still the first line for the treatment of severe falciparum malaria. However, there is an urgent need to test and apply other alternative drugs, especially sulfadoxine-pyrimethamine combination, which is free of side-effects such as hypoglycaemia and preterm labour.

\section{References}

1. Ahmed SM et al. Malaria parasitemia during delivery. Saudi medical journal,

2. Taha TE et al. Levels and determinants of 2001, 23:684-8. perinatal mortality in Central Sudan. In- 
ternational journal of gynaecology and obstetrics, 1994, 45:109-15.

3. Taha TE et al. Malaria and low birth weight in central Sudan. American journal of epidemiology, 1993, 138:318-25.

4. Adam et al. Quinine therapy in severe Plasmodium falciparum malaria during pregnancy in Sudan. Eastern Mediterranean health journal, 2004, 10(1/2): 159-66.

5. Maternal mortality in Sudan. Inter-regional meeting on the prevention of maternal mortality, Geneva 11-15 November, 1985. Geneva, World Health Organization, 1985 (Unpublished document FHE/PMM/85.95).

6. Dafallah SE, El-Agib FH, Bushra GO. Maternal mortality in a teaching hospital in Sudan. Saudi medical journal, 2003, 24:369-73.

7. Dolan $\mathrm{G}$ et al. Bed nets for the prevention of malaria and anaemia in pregnancy. Transactions of the Royal Society of Tropical Medicine and Hygiene, 1993; 87:620-6.

8. Adam I et al. In the Sudan: chloroquine resistance is worsening and quinine resistance is emerging. Sudan medical journal, 2001, 39:1-5.

9. Elkheir HK et al. Efficacy of sulphadoxine and pyrimethamine, doxycycline and their combination in the treatment of chloroquine resistant falciparum malaria. Saudi medical journal, 2001, 228: 690-3.

10. Adam I et al. Efficacy of sulfadoxine-pyrimethamine in the treatment of uncomplicated Plasmodium falciparum malaria in a small sample of Sudanese children. Eastern Mediterranean health journal, 2004, 10(3):309-14.

11. Yousif MA, Adeel AA. Antimalarial prescribing pattern in Gezira State: precepts and practice. Eastern Mediterranean health journal, 2000, 6:939-47.

12. World Health Organization, Division of Control of Tropical Diseases. Severe and complicated malaria. Transactions of the Royal Society of Tropical Medicine and Hygiene, 1990, 84(suppl.2):1-65.

13. Kofoed PE et al. Treatment of Plasmodium falciparum malaria with quinine in Guinea-Bissau: one daily dose is sufficient. Transactions of the Royal Society of Tropical Medicine and Hygiene, 2002, 96:185-8.

14. World Health Organization, Communicable Diseases Cluster. Severe falciparum malaria. Transactions of the Royal Society of Tropical Medicine and Hygiene, 2000, 94(suppl.1):S1-90.

15. Brockman A et al. Application of genetics marker to the identification of recrudescent $P$. falciparum infection on the NorthWestern border of Thailand. American journal of tropical medicine and hygiene, 1999, 60:14-21.

16. Looareesuwan $S$ et al. Quinine and severe falciparum malaria in late pregnancy. Lancet, 1985, 2:4-8.

17. McGready R et al. Quinine and mefloquine in the treatment of multidrug-resistant Plasmodium falciparum malaria in pregnancy. Annals of tropical medicine and parasitology, 1998, 92:643-53.

18. Mukherjee S, Bhose LN. Induction of labour and abortion with quinine infusion in intrauterine fetal deaths. American journal of obstetrics and gynecology, 1968, 101:853-4.

19. Khalil IF. Sensitivity of chloroquine resistant Plasmodium falciparum to fansimef, mefloquine and halofantrine in Gadaref, eastern Sudan [MSc thesis]. Khartoum, Sudan, University of Khartoum, 1995.

لبحلة الصحية لشرق المثتوسط، منظمة الصحة العلمية، البحلد العاشر، العدذان ع-0، ع • • 